\title{
Endometrial pathology in breast cancer patients: Effect of different treatments on ultrasonographic, hysteroscopic and histological findings
}

\author{
MARIA LE DONNE ${ }^{1}$, ANGELA ALIBRANDI ${ }^{2}$, LEONARDA CIANCIMINO $^{1}$, \\ ANDREA AZZERBONI $^{1}$, BENITO CHIOFALO $^{1}$ and ONOFRIO TRIOLO ${ }^{1}$ \\ Departments of ${ }^{1}$ Gynecological, Obstetrical Sciences and Reproductive Medicine and \\ ${ }^{2}$ Economical, Business and Environmental Sciences and Quantitative Methods, University of Messina, Messina, Italy
}

Received October 15, 2012; Accepted January 4, 2013

DOI: $10.3892 / \mathrm{ol} .2013 .1156$

\begin{abstract}
Breast cancer patients have an increased risk of endometrial pathology. To investigate whether the incidence of endometrial abnormalities and their clinicopathological features were affected by receiving tamoxifen (TAM), non-steroidal aromatase inhibitors (AIs) or no treatment (NT), 333 peri/postmenopausal breast cancer patients, who were referred to the Department of Gynecological, Obstetrical Sciences and Reproductive Medicine for gynecological assessment, were reviewed retrospectively. Transvaginal ultrasonographic (TVUS), hysteroscopic and histological findings were investigated. Endometrial histological findings included: atrophy in $61,94.3$ and $55.6 \%$ of cases in the TAM, AIs and NT groups, respectively; polyps in 30.9, 31.4 and $42.2 \%$ of cases in the TAM, AIs and NT groups, respectively; hyperplasia in $3 \%$ of patients in the TAM group and $11.1 \%$ of patients in the NT group; and cancer in $3.8 \%$ of cases in the TAM group and $11.1 \%$ of cases in the NT group. There was a significant correlation between the duration of TAM treatment and the severity of endometrial pathology. In all groups, there was a significant correlation between hysteroscopic and histological findings with regard to the diagnosis of endometrial atrophy, polyps, hyperplasia and cancer $(\mathrm{P}<0.001)$. In conclusion, these data revealed that there was a higher incidence of endometrial pathology in the NT group compared with the TAM group, which was significant for endometrial hyperplasia and cancer. The chance of developing high-risk histological subtypes of endometrial cancer was independent of TAM use. Lastly, although there was no significant difference in recurrent vaginal bleeding
\end{abstract}

Correspondence to: Professor Maria Le Donne, Department of Gynecological, Obstetrical Sciences and Reproductive Medicine, University of Messina, University Hospital 'G. Martino', Via Consolare Valeria, Messina I-98125, Italy

E-mail: marialedonne@tin.it

Key words: endometrial carcinoma, tamoxifen, aromatase inhibitors, transvaginal ultrasound, hysteroscopy and mean endometrial thickness between the TAM and AIs groups, patients receiving AIs did not exhibit hyperplastic, dysplastic or neoplastic changes in the endometrium. This study indicates that breast cancer patients require screening for endometrial pathology; TVUS alone is useful in asymptomatic patients, however, in patients where the endometrial line is irregular or its thickness is $>3 \mathrm{~mm}$, hysteroscopy with directed biopsy is the appropriate diagnostic method.

\section{Introduction}

Breast cancer patients have an increased risk of endometrial pathology. The factors involved are not well established, however it may be linked to a hyperestrogenic state and genetic predisposition.

Tamoxifen (TAM) is widely used as an adjuvant hormonal treatment for females with hormone-sensitive breast cancer. It has been effective in reducing the risk of recurrence and mortality via selective estrogen receptor (ER) modulation which causes ER antagonism in breast tissue (1). However, in the endometrium, TAM has ER agonist activity that is able to stimulate proliferation, causing an increased risk of uterine pathologies, including typical and atypical glandular hyperplasia, endometrial polyps, uterine fibroids, adenocarcinoma and sarcoma (2-9). The risks are highest after long-term use $(8,10)$.

Third generation non-steroidal aromatase inhibitors (AIs), including letrozole and anastrozole, are approved for the treatment of early breast cancer in hormone receptor-positive postmenopausal patients. They decrease estrogen levels by inhibiting the aromatase enzyme, which is responsible for the conversion of androgens to estrogens $(11,12)$. Previous studies have provided evidence which supports the administration of AIs for treating breast cancer, either as 5 years of adjuvant therapy or following 2.5 or 5 years of TAM-based adjuvant therapy as part of a switching strategy $(13,14)$. These had a clinical advantage over TAM treatment (12-14).

Since AIs have no effect on ERs, gynecological symptoms are decreased with AIs treatment compared with TAM treatment; a significant reduction in mean endometrial thickness in patients who switched to anastrozole compared with those who continued on TAM treatment has been observed (13,15-19). 
Table I. Clinical features of the study population.

\begin{tabular}{lcccc}
\hline Variable & TAM & AIs & NT & P-value $^{\mathrm{a}}$ \\
\hline Age (years), mean \pm SD & $59.4 \pm 10.6$ & $59.6 \pm 11.4$ & $60.6 \pm 11.3$ & $\mathrm{~ns}$ \\
Parity, mean \pm SD & $2.3 \pm 1.4$ & $1.9 \pm 1.4$ & $2.4 \pm 1.9$ & $\mathrm{~ns}$ \\
Therapy duration (months), mean \pm SD & $41.1 \pm 33$ & $33.3 \pm 31.4$ & 0 & 0.000 \\
US endometrial thickness (mm), mean \pm SD & $7.7 \pm 7.2$ & $6.0 \pm 7.1$ & $4.8 \pm 7.7$ & 0.009 \\
Vaginal bleeding, $(\%)$ & $58(24.6)$ & $7(20)$ & $14(31.1)$ & $\mathrm{ns}$ \\
Menopause, $\mathrm{n}(\%)$ & $216(91)$ & $32(91.4)$ & $40(88.9)$ & $\mathrm{ns}$ \\
\hline
\end{tabular}

${ }^{a}$ Kruskall-Wallis test. TAM, tamoxifen; AIs, non-steroidal aromatase inhibitors; NT, no treatment; ns, not significant; US, ultrasonography.

Several approaches have been proposed for screening females with breast cancer for abnormal endometrial proliferation or endometrial cancer. Transvaginal ultrasonography (TVUS) has improved the measurement and characterization of the endometrium in a variety of clinical situations. However, reported measurements of endometrial thickness of $>6-8 \mathrm{~mm}$ have subsequently been associated with inactive endometrium following biopsies in TAM-treated patients (20-22). Little benefit has been obtained by the routine use of sonohysterography $(23,24)$. Hysteroscopy with biopsy is the most sensitive and specific method for obtaining a reliable diagnosis, however it is an invasive procedure $(9,15,25)$.

The aim of this study was to investigate whether the incidence of endometrial abnormalities and their clinicopathological features in peri/postmenopausal breast cancer patients, who were referred to the Department of Gynecological, Obstetrical Sciences and Reproductive Medicine for gynecological assessment, were affected by receiving no treatment (NT), TAM or AIs. A further aim of the study was to determine the best approach for screening these patients for endometrial pathology.

\section{Patients and methods}

Patients. Between January 2002 and December 2011, 333 peri/postmenopausal females, who underwent surgery for invasive breast cancer and had suspected endometrial changes (abnormal vaginal bleeding and/or thickened endometrium), were referred to the Department of Gynecological, Obstetrical Sciences and Reproductive Medicine for gynecological assessment and were reviewed retrospectively. The patients had not received hormone therapy or had gynecological pathology prior to the diagnosis of breast cancer. The study was approved by the Ethics Committee of the University of Messina, Messina, Italy. Written informed consent was obtained from the patients.

Treatment. TAM was administered to 242 patients (TAM group) at a daily dose of $20 \mathrm{mg}$, with the exception of two patients who received a daily dose of $40 \mathrm{mg}$ and two patients who received a daily dose of $60 \mathrm{mg}$. AIs were administered to 36 patients (AIs group) at a daily dose of $2.5 \mathrm{mg}$ and 55 patients did not receive TAM or AIs treatment due to having a hormone receptor-negative status (NT group).
TVUS evaluation. TVUS was performed using a $5.0-\mathrm{MHz}$ Aloka 500 system. The largest endometrial thickness was recorded on the midline sagittal scan by including the double layer of the endometrium.

Hysteroscopy. Hysteroscopy was performed by the same operator using a 5, 3 or $1.9 \mathrm{~mm}, 30$-degree optical system (Karl Storz, Tuttningen, Germany) without anesthesia. Sorbitol solution (5\% glucose) or 5\% mannitol solution was used for uterine distension, with an insufflator that maintained a $100-150-\mathrm{mmHg}$ pressure in the uterine cavity. All patients underwent targeted biopsy during the same procedure and the material was sent for pathological examination.

Statistical analysis. Numerical variables are expressed as the mean \pm standard deviation and the categorical variables are expressed as a number and percentage. A non-parametric approach was used since the examined variables were not normally distributed, as verified by the Kolmogorov-Smirnov test.

Spearman's rank correlation coefficient was applied to evaluate the interdependence between vaginal bleeding and/or treatment duration (in months) and TVUS, hysteroscopic and histological features for each group. The same test was applied to evaluate the overall correlation between TVUS, hysteroscopic and histological findings. The Kruskall-Wallis test was applied to assess the existence of significant differences between the three groups with regard to all clinical, TVUS, hysteroscopic and histological features. The Mann-Whitney U test was applied to perform a two-by-two comparison between the groups.

$\mathrm{P}<0.05$ was considered to indicate a statistically significant difference. The software used for statistical analysis was SPPS for Windows 11.0 (2001; SPSS, Inc., Chicago, IL, USA).

\section{Results}

General patient data. Only 316 of 333 peri/postmenopausal breast cancer patients were included in this study; 3 patients were withdrawn due to breast cancer recurrence, 6 patients were withdrawn due to hysteroscopy failure as a result of cervical stenosis and in 8 cases, the amount of endometrial material was insufficient for histological diagnosis.

Clinical features. Clinical features of the study population are shown in Table I. There was no significant difference between 
Table II. Ultrasonographic, hysteroscopic and histological features.

\begin{tabular}{|c|c|c|c|c|}
\hline Variable & $\begin{array}{l}\text { TAM, } n(\%) \\
\text { Total } n=236\end{array}$ & $\begin{array}{l}\text { AIs, n }(\%) \\
\text { Total } n=35\end{array}$ & $\begin{array}{l}\mathrm{NT}, \mathrm{n}(\%) \\
\text { Total } \mathrm{n}=45\end{array}$ & P-value ${ }^{a}$ \\
\hline \multicolumn{5}{|l|}{ Ultrasonography } \\
\hline $5-8 \mathrm{~mm}$ & $34(14.4)$ & $3(8.6)$ & $5(11.1)$ & ns \\
\hline$>8 \mathrm{~mm}$ & $111(47)$ & $16(45.7)$ & $12(26.7)$ & ns \\
\hline Intracavitary fluid & $26(11)$ & $1(2.9)$ & $3(6.7)$ & ns \\
\hline Endometrial lesion & $33(14)$ & $9(25.7)$ & $13(28.9)$ & 0.021 \\
\hline \multicolumn{5}{|l|}{ Hysteroscopy } \\
\hline Atrophy & $216(91.5)$ & $34(97.1)$ & $34(75.6)$ & 0.002 \\
\hline Polyp & 91 (38.6) & $12(34.3)$ & $16(35.6)$ & ns \\
\hline Hyperplasia & $17(7.2)$ & 0 & $5(11.1)$ & ns \\
\hline Suspected cancer & 7 (3) & 0 & $4(8.9)$ & ns \\
\hline \multicolumn{5}{|l|}{ Histology } \\
\hline Atrophy & $144(61)$ & $33(94.3)$ & $25(55.6)$ & $<0.001$ \\
\hline Polyp & 73 (30.9) & $11(31.4)$ & $19(42.2)$ & ns \\
\hline Hyperplasia & $7(3)$ & 0 & $5(11.1)$ & 0.015 \\
\hline Dysplasia & $5(2.1)$ & 0 & 0 & $\mathrm{~ns}$ \\
\hline Cancer & $9(3.8)$ & 0 & $5(11.1)$ & 0.038 \\
\hline
\end{tabular}

${ }^{a}$ Kruskall-Wallis test. TAM, tamoxifen; AIs, non-steroidal aromatase inhibitors; NT, no treatment; ns, not significant.

the three groups with regard to age, parity, menopausal status and incidence of vaginal bleeding. The age of the patients ranged from 45 to 91 years, with a mean of 59.6 years. All patients were multiparas and $91.1 \%$ were postmenopausal.

The mean duration of the TAM and AIs treatment was 41.1 and 33.3 months, respectively.

The mean endometrial thickness, as evaluated by TVUS was $7.7 \mathrm{~mm}$ in the TAM group, $6 \mathrm{~mm}$ in the AIs group and $4.8 \mathrm{~mm}$ in the NT group. Despite no significant difference being identified between the TAM group and the AIs group $(\mathrm{P}=0.154$; Mann-Whitney $\mathrm{U}$ test $)$, there was a significant difference between the 3 groups $(\mathrm{P}=0.009$; Kruskall-Wallis test).

TVUS, hysteroscopic and histological findings. The TVUS, hysteroscopic and histological findings are summarized in Table II. The mean endometrial thickness, as visualized by TVUS, was $5-8 \mathrm{~mm}$ in $14.4,8.6$ and $11 \%$ of cases and $>8 \mathrm{~mm}$ in $47,45.7$ and $26.7 \%$ of cases in the TAM, AIs and NT groups, respectively; no significant differences were identified between the three groups.

Intracavitary fluid was revealed by TVUS in 11, 2.9 and $6.7 \%$ of patients in the TAM, AIs and NT groups, respectively, and there was no significant difference between the three groups. TVUS identified an endometrial lesion in 14, 25.7 and $28.9 \%$ of cases in the TAM, AIs and NT groups, respectively.

Hysteroscopy revealed that polyps were present in 38.6, 34.3 and $35.6 \%$ of patients and cystic atrophy was concurrently detected in $35.5,31.4$ and $31.1 \%$ of patients in the TAM, AIs and NT groups, respectively.

Endometrial atrophy alone was visualized in $13.5 \%$ of patients in the TAM group, $62.8 \%$ of patients in the AIs group and $44.4 \%$ of patients in the NT group. Hyperplastic lesion was diagnosed in $7.2 \%$ of cases in the TAM group and $11.1 \%$ of cases in the NT group. Neoplastic lesion was suspected in $3 \%$ of cases in the TAM group and $8.9 \%$ of cases in the NT group.

Endometrial histological findings included: atrophy in $61,94.3$ and $55.6 \%$ of cases and polyps in 30.9, 31.4 and $42.2 \%$ of cases in the TAM, AIs and NT groups, respectively; hyperplasia in $3 \%$ of patients in the TAM group and $11.1 \%$ of patients in the NT group; and cancer in $3.8 \%$ of cases in the TAM group and $11.1 \%$ of cases in the NT group.

One case of cervical carcinoma and one of gastric carcinoma were also diagnosed in the TAM group.

Significant differences were identified between the 3 groups with regard to the incidence of histological diagnosis of atrophy, hyperplasia and endometrial cancer $(\mathrm{P}<0.001$, $\mathrm{P}=0.015$ and $\mathrm{P}=0.038$, respectively). However, hyperplasia, dysplasia and endometrial cancer did not occurr in the AIs group. Furthermore, the incidence of endometrial cancer in the NT group $(11.1 \%)$ was significantly higher than in the TAM group (3.8\%; $\mathrm{P}=0.038)$.

Of the 14 endometrial cancer cases observed, although the main histotype was found adenocarcinoma, including endometrioid carcinoma, six cases $(42.8 \%)$ were high risk histological subtypes, including three papillary serous carcinomas (one case in the TAM group and two in the NT group), two mesodermal mixed tumors in the TAM group and one sarcoma in the NT group.

Amongst patients treated with TAM, there was a significant correlation between the therapy duration and the presence of intracavitary lesion or fluid, as identified by TVUS $(\mathrm{P}<0.001)$. No correlation existed between the therapy duration and vaginal bleeding or endometrial thickness. 
Table III. Correlation between the ultrasonographic and hysteroscopic findings.

\begin{tabular}{lcccc}
\hline Characteristic & Atrophy & Polyp & Hyperplasia & Suspected cancer \\
\hline Endometrial thickness & $\mathrm{ns}$ & $\mathrm{ns}$ & $\mathrm{ns}$ & $\mathrm{ns}$ \\
Intracavitary lesion & $\mathrm{ns}$ & 0.01 & $\mathrm{~ns}$ & $\mathrm{~ns}$ \\
Intracavitary fluid & $\mathrm{ns}$ & 0.013 & $\mathrm{~ns}$ & $\mathrm{~ns}$ \\
\hline
\end{tabular}

ns, not significant.

Table IV. Correlation between the ultrasonographic and histological findings.

\begin{tabular}{lccccc}
\hline Characteristic & Atrophy & Polyp & Hyperplasia & Dysplasia & Cancer \\
\hline Endometrial thickness & $\mathrm{ns}$ & $\mathrm{ns}$ & $\mathrm{ns}$ & $\mathrm{ns}$ & $\mathrm{ns}$ \\
Intracavitary lesion & 0.012 & 0.001 & $\mathrm{~ns}$ & $\mathrm{~ns}$ & $\mathrm{~ns}$ \\
Intracavitary fluid & $\mathrm{ns}$ & 0.049 & $\mathrm{~ns}$ & $\mathrm{~ns}$ \\
\hline
\end{tabular}

ns, not significant.

Table V. Correlation between the hysteroscopic and histological findings.

\begin{tabular}{lccccc}
\hline Variable & Atrophy & Polyp & Hyperplasia & Dysplasia & Cancer \\
\hline Atrophy & $<0.001$ & $\mathrm{~ns}$ & $<0.001$ & $\mathrm{~ns}$ & $<0.001$ \\
Polyp & $\mathrm{ns}$ & $<0.001$ & $\mathrm{~ns}$ & $\mathrm{~ns}$ & $\mathrm{~ns}$ \\
Hyperplasia & 0.02 & $\mathrm{~ns}$ & $<0.001$ & $\mathrm{~ns}$ & $\mathrm{~ns}$ \\
Suspected cancer & $<0.001$ & 0.019 & $\mathrm{~ns}$ & 0.042 & $<0.001$ \\
\hline
\end{tabular}

ns, not significant.

The length of TAM treatment was also correlated with hysteroscopic identification of suspected cancer $(\mathrm{P}=0.009)$ and histological diagnosis of endometrial dysplastic or neoplastic lesions ( $\mathrm{P}=0.009$ and $\mathrm{P}=0.015$, respectively). The three documented endometrial cancer histotypes with a poor prognosis in TAM group, one papillary serous carcinoma and two mesodermal mixed tumors, were not correlated with the dosage and occurred after 72, 60 and 160 months of therapy, respectively.

Among patients treated with AIs, there was no significant correlation between therapy duration and vaginal bleeding or TVUS, hysteroscopic and histological findings.

Vaginal bleeding was present in 24.4 and $20 \%$ of patients in the TAM and AIs groups, respectively. In the TAM group, vaginal bleeding was significantly correlated with endometrial thickness and intracavitary lesion, as identified by TVUS $(\mathrm{P}=0.003$ and $\mathrm{P}=0.002$, respectively), with hysteroscopically suspected cancer lesion $(\mathrm{P}=0.003)$ and with histological diagnosis of endometrial dysplasia $(\mathrm{P}=0.003)$. In the AIs group, vaginal bleeding was significantly correlated with hystological diagnosis of endometrial polyp $(\mathrm{P}=0.047)$. In the NT group, vaginal bleeding was significantly correlated with intracavitary lesion, as identified by TVUS.

TVUS identification of intracavitary fluid and intracavitary lesion was significantly correlated with hysteroscopic
( $\mathrm{P}=0.013$ and $\mathrm{P}=0.001$, respectively) and histological $(\mathrm{P}=0.049$ and $\mathrm{P}=0.001$, respectively) diagnosis of endometrial polyp, as shown in Tables III and IV. There was a significant correlation between hysteroscopic and histological findings, with regard to the diagnosis of endometrial atrophy, polyp, hyperplasia and cancer in all groups $(\mathrm{P}<0.001)$, as shown in Table V.

\section{Discussion}

The major systemic treatment for patients with $\mathrm{ER}^{+}$invasive breast cancer is endocrine therapy. Adjuvant therapy with TAM for 5 years reduces the yearly breast cancer mortality rate by $31 \%$ (1).

There is conflicting evidence with regard to the association between an increase in endometrial thickness and the duration of TAM treatment $(6,10,26,27)$. In this study, endometrial thickness was not correlated with the duration of TAM treatment. In agreement with other studies, TAM-induced cystically thickened endometrium revealed by ultrasound is not confirmed by hysteroscopy (atrophic endometrium) in $50-90 \%$ of cases and has histology which corresponds with condensated stroma and fluid-filled, cystically dilated glands lined with flattened epithelium (28). TAM treatment is associ- 
ated with an increased risk of developing endometrial cancer; the relative risk is estimated to be two- to six-fold $(2,5,29)$ and this increases with the duration and the cumulative dose of therapy $(3,8,10,29,30)$.

In our study, the presence of endometrial pathology in $41.9 \%$ of females treated with TAM confirms, in agreement with the current literature, that long-term TAM use in breast cancer patients is associated with a higher incidence of uterine pathology. Several studies have documented a connection between TAM therapy and benign uterine pathologies (endometrial polyps and hyperplastic endometrial changes). Their incidence exceeds by 3, 9 and 10 times the incidence of endometrial cancer in TAM-treated patients (30), suggesting that the majority of the proliferative effects of TAM on endometrium are unlikely to progress to cancer.

There is no accordance between previous studies with regard to the duration of TAM therapy and the incidence of endometrial pathology. In this study, a significant correlation has been demonstrated between the duration of the TAM treatment and the severity of endometrial pathology.

The process by which TAM contributes to the carcinogenesis of endometrial cancer is unknown; the finding of atypical metaplasia in cystic endometrial atrophy is not explained by estrogenic effects, but points to anti-estrogen or -progesterone-like activity (31).

Bergman et al (8) showed that long-term TAM use was positively correlated with p53 overexpression in endometrial tumors and inversely correlated with ER status. p53-positive tumors were often steroid receptor-negative and belonged to a group of malignant mixed mesodermal tumors and endometrial sarcomas (8). Subsequently, Ferguson et al observed that gene expression in these tumors was the same as those that have no association with TAM use (32). Although the ER pathway and its role in tumorigenesis in TAM users is controversial, a more frequent expression of ER- $\beta$ and a lower expression of ER- $\alpha$ was observed by Wilder et al in TAM-associated tumors (33).

Weaknesses of our study include its retrospective design and incomplete information about the hormone status (for example, $\mathrm{ER}^{+-}$), histological subtype and stage of breast cancer.

This study showed that the documented endometrial cancer histotypes that had a poor prognosis were not correlated with the dosage or duration of TAM therapy.

Our data were in agreement with those of Slomovitz et al, which showed that females who developed endometrial cancer following a diagnosis of breast cancer had an increased risk of developing the high-risk histological subtypes, independent of TAM use. No significant differences were identified in the clinical or pathological features of endometrial cancer between TAM users and non-users (34).

On the other hand, Fles et al have demonstrated that tumors (all subtypes) which had developed after prolonged TAM use are not distinguishable from those of non-users on the basis of their genomic profile (35).

Data from this study revealed that there was a higher incidence of endometrial pathology in the NT group than in the TAM group, which was significant for endometrial hyperplasia (11.2 vs. $3 \%$, respectively) and cancer (11.1 vs. $3.8 \%$, respectively). Furthermore, three out of five endometrial cancer cases among TAM non-users were high-risk histological subtypes, including two papillary serous carcinomas and one sarcoma.

These data are in agreement with previous studies which showed that the standardized incidence ratios for uterine cancer in breast cancer patients is increased amongst TAM users and non-users and this may be due to shared etiological risk factors in uterine and breast cancer $(36,37)$.

Patients who were treated for breast cancer had an increased risk of leukemia and gynecological cancer and a slightly enhanced risk of gastrointestinal cancer, in addition to the well-known risk of developing sarcomas and lung cancer after radiotherapy (36-38). In this study, the two mesodermal mixed tumors cases in the TAM group and the sarcoma case in the NT group had undergone radiotherapy.

Adjuvant treatment with AIs has been shown to be more effective than TAM treatment in reducing the risk of recurrence (11-14), with a lower risk of developing endometrial pathology compared with TAM therapy $(13-16,19)$. Furthermore, AIs may reverse TAM-induced endometrial abnormalities (17-20). Treatment with AIs has been shown to significantly reduce the incidence of life-threatening adverse events, including endometrial cancer, compared with TAM treatment (39).

Although no significant differences were identified for recurrent vaginal bleeding and mean endometrial thickness between the TAM and AIs groups, patients in this study who received AIs did not exhibit hyperplastic, dysplastic or neoplastic changes of the endometrium.

Endometrial cancer is a serious adverse event which may occur in breast cancer patients, therefore yearly gynecological assessments and rapid assessment in the occurrence of vaginal bleeding should implemented. TVUS has been reported to be appropriate for screening the endometrium for intrauterine pathology before endocrine treatment in females with breast cancer. An endometrial thickness of $3 \mathrm{~mm}$ is the threshold for requiring further investigation with hysteroscopy and endometrial biopsy (15). However, TVUS is not the best method for assessing TAM-induced endometrial changes, due to the high false-positive rate $(46-56 \%)(9,16,22)$ and there is discordance between TVUS, hysteroscopic and histological findings $(9,20,21)$.

Our data show that TVUS was not accurate in identifying hyperplasia and polyps, as endometrial thickness measurements missed hyperplastic changes and polyps in a large number of cases. Furthermore, for intracavitary lesions, TVUS was unable to differentiate between a polyp, which may contain cancerous cells, and endometrial glandulocystic atrophy. Hysteroscopy is more accurate in the diagnosis of polyps, hyperplastic and neoplastic changes and it is the only method that provides a direct view of the endometrial cavity and the possibility of performing directed biopsies for a definitive diagnosis $(9,25)$.

This study, in accordance with previous studies, indicates that breast cancer patients require endometrial screening, although the cost/efficacy ratio is not favorable. TVUS alone is useful in asymptomatic patients, however, if the endometrial line is irregular or its thickness is $>3 \mathrm{~mm}$, hysteroscopy with directed biopsy is the appropriate diagnostic method $(9,13,15,40)$. 


\section{References}

1. No authors listed: Systemic treatment of early breast cancer by hormonal, cytotoxic, or immune therapy. 133 randomized trials involving 31,000 recurrences and 24,000 deaths among 75,000 women. Early Breast Cancer Trialists' Collaborative Group. Lancet 339: 1-15, 1992.

2. Fisher B, Costantino JP, Redmond CK, et al: Endometrial cancer in tamoxifen-treated breast cancer patients: findings from the National Surgical Adjuvant Breast and Bowel Project (NSABP) B-14. J Natl Cancer Inst 86: 527-537, 1994.

3. Fornander T, Rutqvist LE, Cedermark B, et al: Adjuvant tamoxifen in early breast cancer: occurence of new primary cancers. Lancet 1: 117-120, 1989.

4. Fugh-Berman A and Epstein S: Tamoxifen: disease prevention or disease substitution? Lancet 340: 1143-1145, 1992.

5. Rutqvist LE, Johansson H, Signomklao T, Johansson U, Fornander T and Wilking N: Adjuvant tamoxifen therapy for early stage breast cancer and second primary malignancies. Stockholm Breast Cancer Study Group. J Natl Cancer Inst 87: 645-651, 1995

6. van Leeuwen FE, Benraadt J, Coebergh JW, et al: Risk of endometrial cancer after tamoxifen treatment of breast cancer. Lancet 343: 448-452, 1994.

7. Jones ME, van Leeuwen FE, Hoogendoorn WE, et al: Endometrial cancer survival after breast cancer in relation to tamoxifen treatment: pooled results from three countries. Breast Cancer Res 14: R91, 2012.

8. Bergman L, Beelen ML, Gallee MP, et al: Risk and prognosis of endometrial cancer after tamoxifen for breast cancer. Comprehensive Cancer Centres' ALERT Group. Assessment of Liver and Endometrial cancer risk following Tamoxifen. Lancet 356: 881-887, 2000.

9. Le Donne M, Lentini M, De Meo L, Benedetto V and Mesiti M: Uterine pathologies in patients undergoing tamoxifen therapy for breast cancer: ultrasonographic, hysteroscopic and histological findings. Eur J Gynaecol Oncol 26: 623-626, 2005.

10. Cohen I, Altaras MM, Shapira J, Tepper R, Rosen DJ, Cordoba M, et al: Time-depedent effect of tamoxifen therapy on endometrial pathology in asymptomatic postmenopausal breast cancer patients. Int J Gynecol Pathol 15: 152-157, 1996.

11. Dowsett M, Jones A, Johnston SR, et al: In vivo measurement of aromatase inhibition by letrozole (CGS 20267) in postmenopausal patients with breast cancer. Clin Cancer Res 1: 1511-1515, 1995.

12. Geisler J, King N, Dowsett M, et al: Influence of anastrozole (Arimidex), a selective, non-steroidal aromatase inhibitor, on in vivo aromatisation and plasma oestrogen levels in postmenopausal women with breast cancer. Br J Cancer 74: 1286-1291, 1996.

13. Howell A, Cuzick J, Baum M, et al; ATAC Trialists' Group: Results of the ATAC (Arimidex, Tamoxifen, Alone or in Combination) trial after completion of 5 years' adjuvant treatment for breast cancer. Lancet 365: 60-62, 2005

14. Boccardo F, Rubagotti A, Puntoni M, et al: Switching to anastrozole versus continued tamoxifen treatment of early breast cancer: preliminary results of the Italian Tamoxifen Anastrozole Trial. J Clin Oncol 23: 5138-5147, 2005.

15. Duffy S, Jackson TL, Lansdown M, et al; ATAC Trialists' Group: The ATAC ('Arimidex', Tamoxifen, Alone or in Combination) adjuvant breast cancer trial: baseline endometrial sub-protocol data on the effectiveness of transvaginal ultrasonography and diagnostic hysteroscopy. Hum Reprod 20: 294-301, 2005.

16. Gerber B, Krause A, Reimer T, et al: Anastrozole versus tamoxifen treatment in postmenopausal women with endocrineresponsive breast cancer and tamoxifen-induced endometrial pathology. Clin Cancer Res 12: 1245-1250, 2006.

17. Valenzano Menada M, Costantini S, Moioli M, et al: Evaluation of endometrial thickness in hormone receptor positive early stage breast cancer postmenopausal women switching from adjuvant tamoxifen treatment to anastrozole. Breast 17: 631-636, 2008.

18. Markovitch O, Tepper R, Fishman A, et al: Aromatase inhibitors reverse tamoxifen induced endometrial ultrasonographic changes in postmenopausal breast cancer patients. Breast Cancer Res Treat 101: 185-190, 2007.

19. Morales L, Timmerman D, Neven P, et al: Third generation aromatase inhibitors may prevent endometrial growth and reverse tamoxifen-induced uterine changes in postmenopausal breast cancer patients. Ann Oncol 16: 70-74, 2005.

20. Cohen I, Beyth Y, Azaria R, Flex D, Figer A and Tepper R: Ultrasonographic measurement of endometrial changes following discontinuation of tamoxifen treatment in postmenopausal breast cancer patients. BJOG 107: 1083-1087, 2000.
21. Liedman R, Lindahl B, Andolf E, Willen R, Ingvar C and Ranstam J: Disaccordance between estimation of endometrial thickness as measured by transvaginal ultrasound compared with hysteroscopy and directed biopsy in breast cancer patients treated with tamoxifen. Anticancer Res 20: 4889-4891, 2000

22. Fung MF, Reid A, Faught W, et al: Prospective longitudinal study of ultrasound screening for endometrial abnormalities in women with breast cancer receiving tamoxifen. Gynecol Oncol 91: 154-159, 2003.

23. Bertelli GF, Valenzano M, Costantini S, et al: Limited value of sonohysterography for endometrial screening in asymptomatic, postmenopausal patients treated with tamoxifen. Gynecol Oncol 78: 275-277, 2000

24. Develioğlu OH, Omak M, Bilgin T, Esmer A and Tüfekçi M: The endometrium in asymptomatic breast cancer patients on tamoxifen: value of transvaginal ultrasonography with saline infusion and Doppler flow. Gynecol Oncol 93: 328-335, 2004.

25. Giorda G, Crivellari D, Veronesi A, Perin T, Campagnutta E, Carbone A and Scarabelli C: Comparison of ultrasonography, hysteroscopy, and biopsy in the diagnosis of endometrial lesions in postmenopausal tamoxifen-treated patients. Acta Obstet Gynecol Scand 81: 975-980, 2002.

26. Hann LE, Giess CS, Bach AM, Tao Y, Baum HJ and Barakat RR: Endometrial thickness in tamoxifen-treated patients: correlation with clinical and pathologic findings. AJR Am J Roentgenol 168: 657-661, 1997.

27. No authors listed: Tamoxifen for early breast cancer: an overview of the randomised trials. Early Breast Cancer Trialists Collaborative Group. Lancet 351: 1451-1467, 1998.

28. Mourits MJ, Van der Zee AG, Willemse PH, Ten Hoor KA, Hollema H and De Vries EG: Discrepancy between ultrasonography and hysteroscopy and histology of endometrium in postmenopausal breast cancer patients using tamoxifen. Gynecol Oncol 73: 21-26, 1999

29. Neven $P$ and Vernaeve $H$ : Guidelines for monitoring patients taking tamoxifen treatment. Drug Saf 22: 1-11, 2000.

30. Jordan VC and Assikis VJ: Endometrial carcinoma and tamoxifen: clearing up a controversy. Clin Cancer Res 1: 467-472, 1995.

31. Dallenbach-Hellweg G and Hahn U: Mucinous and clear cell adenocarcinomas of the endometrium in patients receiving antiestrogens (tamoxifen) and gestagens. Int J Gynecol Pathol 14: $7-15,1995$.

32. Ferguson SE, Olshen AB, Viale A, Awtrey CS, Barakat RR and Boyd $\mathrm{J}$ : Gene expression profiling of tamoxifen-associated uterine cancers: evidence for two molecular classes of endometrial carcinoma. Gynecol Oncol 92: 719-725, 2004.

33. Wilder JL, Shajahan S, Khattar NH, et al: Tamoxifen-associated malignant endometrial tumors: pathologic features and expression of hormone receptors estrogen-alpha, estrogen-beta and progesterone; a case controlled study. Gynecol Oncol 92: 553-558, 2004.

34. Slomovitz BM, Sun CC, Ramirez PT, Bodurka DC, Diaz P and Lu KH: Does tamoxifen use affect prognosis in breast cancer patients who develop endometrial cancer? Obstet Gynecol 104: 255-260, 2004

35. Fles R, Hoogendoorn WE, Platteel I, et al: Genomic profile of endometrial tumors depends on morphological subtype, not on tamoxifen exposure. Genes Chromosomes Cancer 49: 699-710, 2010.

36. Schaapveld M, Visser O, Louwman MJ, et al: Risk of new primary nonbreast cancers after breast cancer treatment: a Dutch population-based study. J Clin Oncol 26: 1239-1246, 2008.

37. Kirova YM, De Rycke Y, Gambotti L, Pierga JY, Asselain B and Fourquet A; Institut Curie Breast Cancer Study Group: Second malignancies after breast cancer: the impact of different treatment modalities. Br J Cancer 98: 870-874, 2008.

38. Kirova YM, Gambotti L, De Rycke Y, Vilcoq JR, Asselain B and Fourquet A: Risk of second malignancies after adjuvant radiotherapy for breast cancer: a large-scale, single institution review. Int J Radiat Oncol Biol Phys 68: 359-363, 2007.

39. Winer EP, Hudis C, Burstein HJ, et al: American Society of Clinical Oncology technology assessment on the use of aromatase inhibitors as adjuvant therapy for postmenopausal women with hormone receptor-positive breast cancer: status report 2004. J Clin Oncol 23: 619-629, 2005.

40. Machado F, Rodríguez JR, León JP, Rodríguez JR, Parrilla JJ and Abad L: Tamoxifen and endometrial cancer. Is screening necessary? A review of the literature. Eur J Gynaecol Oncol 26: $257-265,2005$ 